\title{
A THREE DIMENSIONAL FINITE ELEMENT METHOD FOR BIOLOGICAL ACTIVE SOFT TISSUE
}

\author{
FORMULATION IN CYLINDRICAL POLAR COORDINATES *
}

\author{
Christian Bourdarias ${ }^{1}$, Stéphane Gerbi ${ }^{1}$ and Jacques Ohayon ${ }^{2}$
}

\begin{abstract}
A hyperelastic constitutive law, for use in anatomically accurate finite element models of living structures, is suggested for the passive and the active mechanical properties of incompressible biological tissues. This law considers the passive and active states as a same hyperelastic continuum medium, and uses an activation function in order to describe the whole contraction phase. The variational and the FE formulations are also presented, and the FE code has been validated and applied to describe the biomechanical behavior of a thick-walled anisotropic cylinder under different active loading conditions.
\end{abstract}

Mathematics Subject Classification. 65M60, 92C10, 92C50, 74L15, 74S05, 74B20.

\section{INTRODUCTION}

Several numerical models, using finite element analysis, were proposed to simulate the heart continuously during the phases of the cardiac cycle $[3,9,16,18]$. In these studies, two approaches were used to model the living tissue. In both of them, the end-diastolic behavior of the muscle was derived from a passive strainenergy function expressed per unit of volume of the passive zero-stress state. Additionally, an active stress tensor was introduced to simulate the contraction of the biological tissue. The main limitation of the first modeling approach is that no active strain-energy function was used to obtain the active stress tensor, which suggests that the activated living tissue is not viewed as a hyperelastic material. In the second approach an active strain-energy function is introduced but an additional intuitive kinematics transformation of the zerostress state is needed to derive the unloaded active state. This last point corresponds to the main limitation of this second modeling approach. Nevertheless Lin and Yin [7] proposed a continuum approach without any additional kinematics transformation but only for two specific states of the cardiac cycle (passive and maximal active states). Therefore, the purpose of the present work is to propose a new method to model the material law of the living tissue, which avoids the previous limitations and allows to describe continuously the whole cardiac cycle. In addition, the finite element formulation with the proposed law was tested by considering simple cases,

Keywords and phrases. Constitutive law, finite element method, biological tissue, hyperelasticity, nonlinear partial differential equations, anisotropic material.

* This study was supported by grant BQROO from the University of Savoie.

${ }^{1}$ Laboratoire de Mathématiques, Université de Savoie, Savoie Technolac, 73376 Le Bourget du Lac, France.

e-mail: Christian.Bourdarias@univ-savoie.fr, Stephane.Gerbi@univ-savoie.fr

2 Laboratoire TIMC-IMAG, Dynacell, UMR CNRS 5525, Domaine de la Merci, 38706 Grenoble, France.

e-mail: Jacques.Ohayon@univ-savoie.fr

(C) EDP Sciences, SMAI 2003 


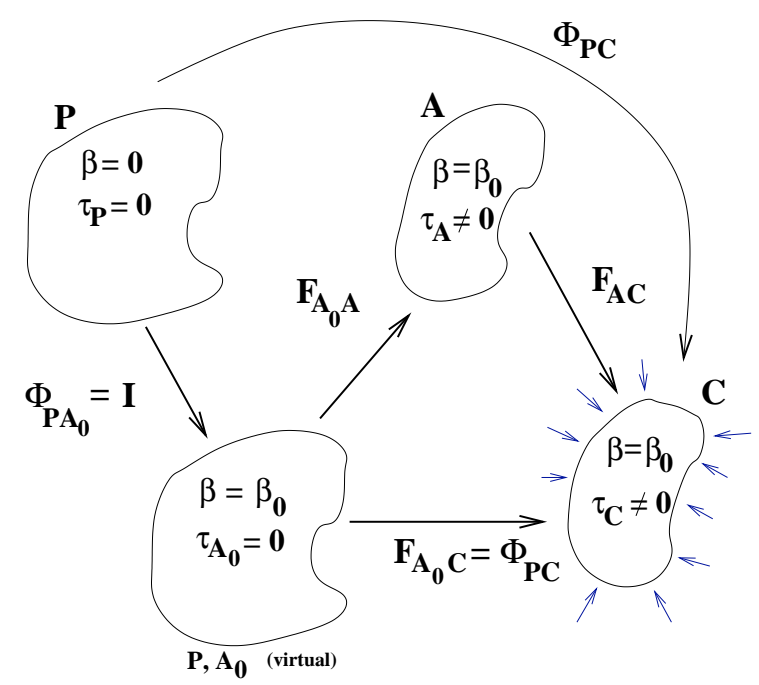

FiguRE 1. Description of the active rheology approach.

which are rectangular samples under different boundary conditions, as well as a finite thick-walled cylinder submitted to an internal pressure.

\section{Mechanical model and finite Element Formulation}

\subsection{Constitutive law for the active biological tissue}

To be consistent with our mathematical formulation, the letter $\boldsymbol{\Phi}$ is used for non elastic gradient tensors and the letter $\mathbf{F}$ is used for elastic gradient tensors. The activation of the muscle fibers changes the properties of the material and at the same time contracts the muscle itself. To have a continuous elastic description during the activation of the tissue, we use an approach similar to the one proposed by Ohayon and Chadwick [12], Taber [16], Lin and Yin [7]. From its passive zero-stress state $P$, the activation of the muscle fibers is modeled by two transformations (Fig. 1). The first one (from state $P$ to virtual state $A_{0}$ ) changes the material properties without changing the geometry, and the second one (from $A_{0}$ to $A$ ) contracts the muscle without changing the properties of the material. Thus, the former is not an elastic deformation and is described by the gradient tensor $\boldsymbol{\Phi}_{P A_{0}}=\mathbf{I}$ where $\mathbf{I}$ is the identity matrix. In this first transformation, only the strain energy function changing the rheology is modified using a time-dependent activation function $\beta(t)(0 \leq \beta(t) \leq 1)$. The second transformation is an elastic deformation caused by the active tension delivered by the fibers and is described by the gradient tensor $\mathbf{F}_{A_{0} A}$. Finally, external loads are applied to state $A$ deforming the body through $\mathbf{F}_{A C}$ into $C$. Thus the global transformation from state $P$ to state $C$ is a non elastic transformation $\left(\boldsymbol{\Phi}_{P C}=\mathbf{F}_{A_{0} C} \boldsymbol{\Phi}_{P A_{0}}\right)$, but can be treated mathematically as an elastic one because $\boldsymbol{\Phi}_{P C}=\mathbf{F}_{A_{0} C}$. The change of the material properties during the activation is described by a time-dependent strain-energy function per unit volume of state $P$ noted $W\left(\mathbf{E}_{P H}, t\right)$ :

$$
W\left(\mathbf{E}_{P H}, t\right)=W_{\mathrm{pas}}\left(\mathbf{E}_{P H}\right)+\beta(t) W_{\mathrm{act}}^{f}\left(\mathbf{E}_{P H}\right),
$$

where $\mathbf{E}_{P H}$ is the Green strain tensor at an arbitrary state $H$ calculated from the zero strain state $P$ (the state $H$ could be one of the states $A_{0}, A$ or $C$ shown in Fig. 1), $W_{\text {pas }}$ represents the contribution of the surrounding collagen matrix and of the passive fiber components, $W_{\text {act }}^{f}$ arises from the active component of the embedded muscle fibers. The last term of the right hand side of the equation gives the variation of the mechanical muscle fibers properties during the activation. We treat the medium as a homogeneous, incompressible and hyperelastic material transversely isotropic with respect to the local muscle fiber direction. This direction is characterized in 


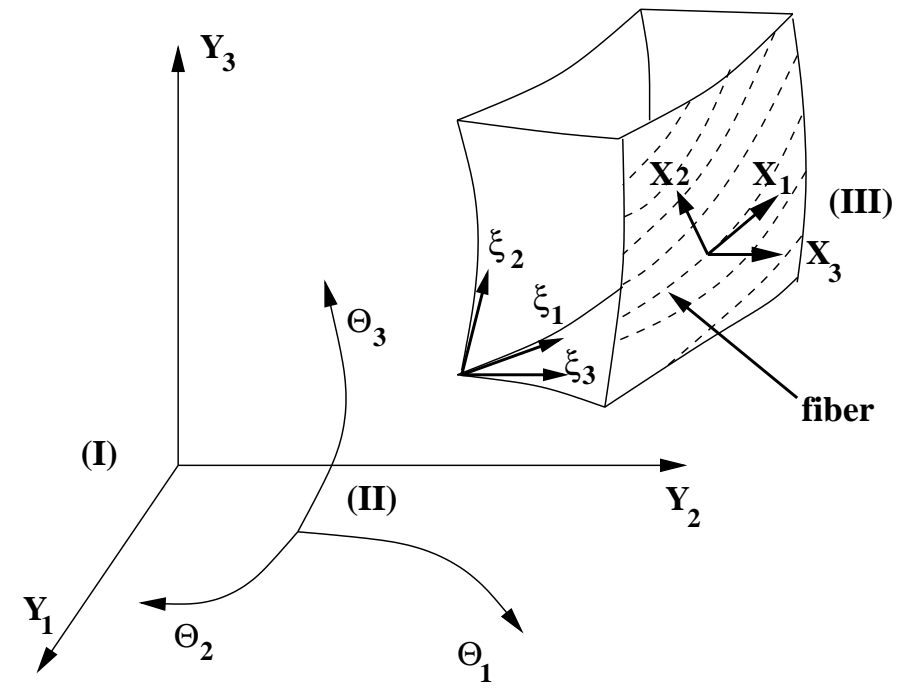

Figure 2. Coordinate systems (adapted from Costa et al. [5]).

an arbitrary state $H$ by the unit vector $\mathbf{f}_{H}$. To incorporate the active contraction, an active fiber stress $T^{(0)}$ is applied in the deformed fiber direction $\mathbf{f}_{C}$, defined by $\mathbf{f}_{C}=\boldsymbol{\Phi}_{P C} \mathbf{f}_{P} /\left\|\boldsymbol{\Phi}_{P C} \mathbf{f}_{P}\right\|$. Hence the Cauchy stress tensor in state $C$ (noted $\tau_{C}$ ) is given by

$$
\boldsymbol{\tau}_{C}=-p_{C} \mathbf{I}+\boldsymbol{\Phi}_{P C} \frac{\partial W\left(\mathbf{E}_{P C}, t\right)}{\partial \mathbf{E}_{P C}} \boldsymbol{\Phi}_{P C}^{T}+\beta(t) T^{(0)} \mathbf{f}_{C} \otimes \mathbf{f}_{C},
$$

where $p_{C}$ is the Lagrangian multiplier resulting of the incompressibility of the material, equivalent to an internal pressure, and the symbol $\otimes$ denotes the tensor product. Notice that the activation function $\beta(t)$ allows us to describe continuously the phases of the cardiac cycle.

\subsection{Variational formulation}

The undeformed body state $P$ consists of a volume $V$ bounded by a closed surface $\mathcal{A}$, and the deformed body state is, as before, noted $C$. The corresponding position vectors, in cartesian basis unit vectors (Fig. 2), are respectively $\mathbf{R}=Y^{R} \mathbf{e}_{R}$ and $\mathbf{r}=y^{r} \mathbf{e}_{r}$. However, we write the equations with suitable curvilinear systems of world coordinates noted $\Theta^{A}$ in the reference configuration (state $P$ ) and $\theta^{\alpha}$ in the deformed configuration (state $C$ ). In this paper we use the same conventional notations (Tab. 1) for vectors, tensors and coordinates systems as Costa et al. [5], where:

- Capital letters are used for coordinates and indices of tensor components associated with state $P$, and lower case letters are related to state $C$.

- $\mathbf{G}$ and $\mathbf{g}$ are the basis vectors in states $P$ and $C$, respectively, for which parenthetical superscript (always a small letter) indicates the associated coordinate system (for example $\mathbf{G}_{I}^{(x)}=\partial \mathbf{R} / \partial X^{I}=\mathbf{R}_{, I}^{(x)}$ and $\left.\mathbf{g}_{i}^{(x)}=\partial \mathbf{r} / \partial x^{i}=\mathbf{r}_{, i}^{(x)}\right)$

Moreover the usual summation convention for repeated indices is used. The Lagrangian formulation of the virtual works principle is given by $[5,8]$

$$
\int_{V} P^{I J} \Phi_{J}^{\cdot \alpha} \nabla_{I}\left(\delta u_{\alpha}\right) \mathrm{d} V=\int_{V} \rho\left(b^{\alpha}-\gamma^{\alpha}\right) \delta u_{\alpha} \mathrm{d} V+\int_{A_{2}} \mathbf{s} . \delta \mathbf{u} \mathrm{d} A,
$$


TABLE 1. Notations for the coordinate systems used to formulate the finite element method (adapted from Costa et al. [5]). (I) Rectangular cartesian reference coordinates, (II) curvilinear world coordinates, (III) normalized finite element coordinate, (IV) locally orthonormal body/fiber coordinates (adapted from Costa et al. [5]).

\begin{tabular}{|c|c|c|c|c|c|c|c|}
\hline & State & Indices & Coord. & $\begin{array}{c}\text { Covariant } \\
\text { basis vectors }\end{array}$ & $\begin{array}{c}\text { Contravariant } \\
\text { basis vectors }\end{array}$ & \multicolumn{2}{|c|}{ Metric tensors } \\
\hline \multirow{2}{*}{ (I) } & $P$ & $R, S$ & $Y^{R}$ & $\mathbf{e}_{R}$ & $\mathbf{e}_{R}$ & $\delta_{R S}$ & $\delta^{R S}$ \\
\hline & $C$ & $r, s$ & $y^{r}$ & $\mathbf{e}_{r}$ & $\mathbf{e}_{r}$ & $\delta_{r s}$ & $\delta^{r s}$ \\
\hline \multirow{2}{*}{ (II) } & $P$ & $A, B$ & $\Theta^{A}$ & $\mathbf{G}_{A}^{(\theta)}=\frac{\partial \mathbf{R}}{\partial \Theta^{A}}$ & $\mathbf{G}^{(\theta) A}$ & $G_{A B}^{(\theta)}$ & $G^{(\theta) A B}$ \\
\hline & $C$ & $\alpha, \beta$ & $\theta^{\alpha}$ & $\mathbf{g}_{\alpha}^{(\theta)}=\frac{\partial \mathbf{r}}{\partial \theta^{\alpha}}$ & $\mathbf{g}^{(\theta) \alpha}$ & $g_{\alpha \beta}^{(\theta)}$ & $g^{(\theta) \alpha \beta}$ \\
\hline (III) & $P$ & $K, L$ & $\xi^{K}$ & $\mathbf{G}_{K}^{(\xi)}=\frac{\partial \mathbf{R}}{\partial \xi^{K}}$ & $\mathbf{G}^{(\xi) K}$ & $G_{K L}^{(\xi)}$ & $G^{(\xi) K L}$ \\
\hline & $P$ & $I, J$ & $X^{I}$ & $\mathbf{G}_{I}^{(x)}=\frac{\partial \mathbf{R}}{\partial X^{I}}$ & $\mathbf{G}^{(x) I}$ & $G_{I J}^{(x)}=\delta_{I J}$ & $G^{(x) I J}=\delta^{I J}$ \\
\hline & $C$ & & & $\mathbf{g}_{I}^{(x)}=\frac{\partial \mathbf{r}}{\partial X^{I}}$ & $\mathbf{g}^{(x) I}$ & $g_{I J}^{(x)}$ & $g^{(x) I J}$ \\
\hline
\end{tabular}

where $P^{I J}$ are the components of the second Piola-Kirchhoff stress tensor $\mathbf{P}$ referred to the basis tensor $\mathbf{G}_{I}^{(x)} \otimes \mathbf{G}_{J}^{(x)}, \Phi_{I}^{\cdot \alpha}=\partial \theta^{\alpha} / \partial X^{I}$ are the components of the gradient tensor $\mathbf{\Phi}_{P C}$ in the basis tensor $\mathbf{g}_{\alpha}^{(\theta)} \otimes \mathbf{G}^{(x) I}$, $\boldsymbol{\delta} \mathbf{u}=\delta u_{\alpha} \mathbf{g}^{(\theta) \alpha}$ is an arbitrary admissible displacement vector, $\nabla_{I}\left(\delta u_{\alpha}\right)=\partial \delta u_{\alpha} / \partial X^{I}-\mathbf{g}_{\alpha, I}^{(\theta)} \cdot \mathbf{g}^{(\theta) \beta} \delta u_{\beta}$ are the components of the covariant differentiation vector $\delta \mathbf{u}$ in the basis vectors $\mathbf{g}^{(\theta) \alpha}\left(\right.$ i.e. $\left.\nabla_{I}(\delta u)=\nabla_{I}\left(\delta u_{\alpha}\right) \mathbf{g}^{(\theta) \alpha}\right)$. The previous differentiation is done with respect to the locally orthonormal body coordinates $\left(X^{I}, I=1,2,3\right)$ of which $X^{1}$ coincides with the local muscle fiber direction. The material density in the undeformed body state $P$ is $\rho, \mathbf{b}=b^{\alpha} \mathbf{g}_{\alpha}^{(\theta)}$ is the body force vector per unit mass, $\gamma=\gamma^{\alpha} \mathbf{g}_{\alpha}^{(\theta)}$ is the acceleration vector, $\mathbf{s}$ is the surface traction per unit area of $\mathcal{A}$, and $A_{2}$ is the part of $\mathcal{A}$ not subject to displacement boundary conditions. The Lagrangian formulation for incompressibility is given by

$$
\int_{V}\left(\operatorname{det} g_{I J}^{(x)}-1\right) p^{*} \mathrm{~d} V=0,
$$

where the metric tensor $g_{I J}^{(x)}$ is defined in Table 1 , and $p^{*}$ is an arbitrary admissible pressure. Equations $((3)$-(4)) represent the variational formulation of a system of nonlinear partial differential equations. For an incompressible medium ( $\operatorname{det} \boldsymbol{\Phi}_{P C}=1$ ), the relation between the second Piola-Kirchoff stress tensor $\mathbf{P}$ and the Cauchy stress tensor $\boldsymbol{\tau}_{C}$ is [8]

$$
\mathbf{P}=\boldsymbol{\Phi}_{P C}^{-1} \cdot \boldsymbol{\tau}_{C} \cdot\left(\boldsymbol{\Phi}_{P C}^{-1}\right)^{T}
$$


We give a complete expression of the components of $\mathbf{P}$ in Appendix A. The surface traction per unit of undeformed area of $\mathcal{A}, \mathbf{s}=s^{\alpha} \mathbf{g}_{\alpha}^{(\theta)}$, is a known boundary loading which could be written using physical Cauchy stress (see Appendix B).

\subsection{Finite element approximation}

Throughout this paper we use a three dimensional finite element with Lagrange trilinear interpolation for the displacements and uniform pressure to compute an approximate solution of equations ((3)-(4)). This element is commonly used and is relevant for the finite element approximation of this type of problem where an incompressibility constraint must be satisfied $[4,6,11,14]$. We neglect the acceleration and body forces $(\mathbf{b}=0$, $\gamma=0)$.

Let $\left(\xi_{K}\right)$ be the Lagrangian normalized finite element coordinates (Fig. 2), the deformed geometric coordinates $\theta^{\alpha}$ in element $e$ are interpolated as

$$
\theta^{\alpha}=\sum_{n(e)=1}^{8} \psi_{n(e)}\left(\xi_{1}, \xi_{2}, \xi_{3}\right) \theta_{n(e)}^{\alpha},
$$

where $\psi_{n(e)}$ is the basis function associated with the local node $n(e)$ and $\theta_{n(e)}^{\alpha}$ is the $\alpha$-coordinate of the local node $n$ of element $e$. Let $\Omega_{\Delta}^{n(e)}$ be the connectivity matrix defined by

$$
\Omega_{\Delta}^{n(e)}=\left\{\begin{array}{lll}
1 & \text { if } & \Delta(n(e), e)=\Delta \\
0 & \text { otherwise }
\end{array}\right.
$$

where $\Delta(p, e)$ is the global node corresponding with the local node $p$ of the element $e$. Then the FE approximation of equations $((3)-(4))$ is

$$
\begin{gathered}
\sum_{e} \sum_{n(e)=1}^{8} \Omega_{\Delta}^{n(e)} \int_{V_{e}} P^{I J}\left[\Phi_{J}^{\cdot \alpha}\left(\psi_{n(e)}\right)_{, I}-\Phi_{J}^{\cdot \beta} \Gamma_{\beta I}^{\alpha} \psi_{n(e)}\right] \mathrm{d} V=\sum_{e} \sum_{n(e)=1}^{8} \Omega_{\Delta}^{n(e)} \int_{A_{2_{e}}} s^{\alpha} \psi_{n(e)} \mathrm{d} A, \\
\int_{V_{e}}\left(\operatorname{det} g_{I J}^{(x)}-1\right) \mathrm{d} V=0,
\end{gathered}
$$

with $\Delta=1, \cdots, \Delta_{\max }, \alpha=1,2,3, \Gamma_{\beta I}^{\alpha}=-\mathbf{g}_{\alpha, I}^{(\theta)} \cdot \mathbf{g}^{(\theta) \beta}$, and where $A_{2_{e}}$ is the part of $A_{e}$ (boundary of element $e$ ) non subject to displacement conditions.

In the case of the loading of a 3D cylindrical sample of a soft tissue with $\xi_{1}$ as fiber direction, we give in Appendix $\mathrm{C}$ a complete detailed algebraic computation of the $\mathrm{FE}$ approximation using trilinear Lagrange interpolation for displacements, and constant approximation $p_{C}(e)$ on each element $e$ of domain $V_{e}$.

\subsection{Finite element solution method}

Let us firstly mention that the unknowns $\left(\theta_{\Delta}^{\alpha}, p_{C}(e)\right), \alpha=1,2,3, \Delta=1, \ldots, \Delta_{\max }, e=1, \ldots, e_{\max },\left(e_{\max }\right.$ is the total number of elements involved in the mesh), are the solution of the nonlinear system of equations ((8)-(9)). To solve this sytem we use the Powell method [13]. This method is a quasi Newton method which consists in: (i) computing the jacobian matrix of an iterate by forward differences (with step $\mathrm{h}=10^{-8}$ ) and (ii) searching the new iterate on a steepest descent line of the jacobian by the so called "dogleg method" [19]. For this sake, we use the free package MINPACK [10]. Moreover, one can observe that in equations ((8)-(9)), the nonlinear functions involve $3 \mathrm{D}$ and $2 \mathrm{D}$ integrals over a rectangular domain. Thus, we use the adaptative gaussian quadrature method to evaluate these integrals with high precision (up to $10^{-12}$ ). For this purpose, we use the free package DCUHRE [2]. The developed numerical code has been called Samuel for "Solid Active MUscle Element" and has been written in Fortran 77 on Personal Computer under LinuX operating system. 


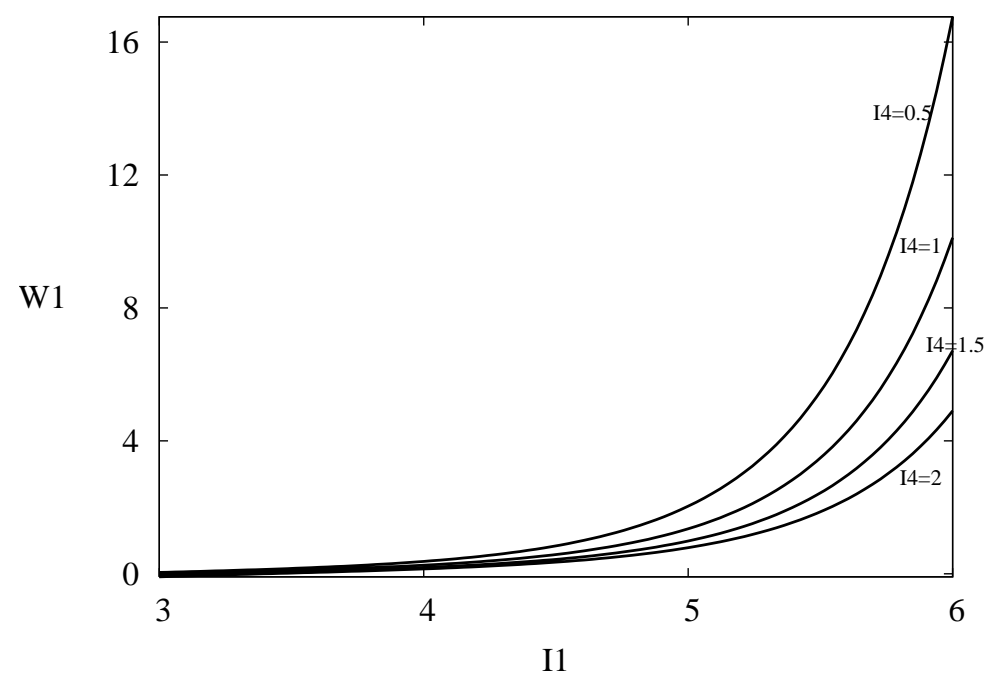

FIGURE 3. First derivative $W_{1}$ of the passive strain-energy function of Lin and Yin with respect to the first strain invariant $I_{1}$ : $W_{1}$ versus $I_{1}$ for $I_{4}=0.5, I_{4}=1, I_{4}=1.5$, and $I_{4}=2$.

\section{Results And Discussion}

\subsection{Loading of an active thin sample of myocardium}

To check the consistency and the accuracy of the proposed FE formulation, we simulate the loading of a thin sample of myocardium $\left(1.0 \times 1.0 \times 0.1 \mathrm{~cm}^{3}\right)$ in which the fibers are uniformly oriented in one direction $\left(Y_{1}\right)$. For these computations, we modified the strain-energy function suggested by Lin and Yin [7] by suppressing their beating term and by introducing our active tension $\beta(t) T^{(0)}$ :

$$
\begin{aligned}
& W_{\mathrm{pas}}\left(\mathbf{E}_{P H}\right)=C_{1}^{p}\left(e^{Q}-1\right) \quad \text { with } \quad Q=C_{2}^{p}\left(I_{1}-3\right)^{2}+C_{3}^{p}\left(I_{1}-3\right)\left(I_{4}-1\right)+C_{4}^{p}\left(I_{4}-1\right)^{2}, \\
& W_{\text {act }}^{f}\left(\mathbf{E}_{P H}\right)=C_{1}^{a}\left(I_{1}-3\right)\left(I_{4}-1\right)+C_{2}^{a}\left(I_{1}-3\right)^{2}+C_{3}^{a}\left(I_{4}-1\right)^{2}+C_{4}^{a}\left(I_{1}-3\right),
\end{aligned}
$$

where $\left(C_{i}^{p}, \quad i=1, \cdots, 4\right)$ and $\left(C_{i}^{a}, \quad i=1, \cdots, 4\right)$ are material constants and $I_{1}, I_{4}$ are two strain invariants given by $I_{1}\left(\mathbf{E}_{P H}\right)=\operatorname{tr} \mathbf{C}_{P H}$ and $I_{4}\left(\mathbf{E}_{P H}\right)=\mathbf{f}_{P} \cdot \mathbf{C}_{P H} \mathbf{f}_{P}$ where $\mathbf{C}_{P H}$ is the right Cauchy-Green strain tensor $\left(\mathbf{C}_{P H}=2 \mathbf{E}_{P H}+\mathbf{I}\right)$. The strain invariant $I_{4}$ is directly related to the fiber extension $\lambda_{f}\left(I_{4}=\lambda_{f}^{2}\right)$. Notice that under the assumptions of incompressibility and transversal isotropy, the strain-energy function $W$ can be expressed in terms of the two strain invariants $I_{1}$ and $I_{4}$ only. Moreover this function satisfies the zero-stress conditions for the passive muscle free of loading. In fact, in this situation, we have $I_{1}=3, I_{4}=1, \beta=0$ and $\partial W_{\text {pas }} / \partial I_{1}=\partial W_{\text {pas }} / \partial I_{4}=0$. The passive anisotropy of the material is illustrated in Figure 3 where we represent the variations of the derivative $W 1=\partial W_{\text {pas }} / \partial I_{1}$ with respect to $I_{1}$ for several values of $I_{4}$. In the isotropic case, this function should depend only upon $I_{1}$ (this case corresponds to the curve labelled " $I_{4}=1$ ").

The coefficients involved in the strain-energy function are those of Lin and Yin [7]: $C_{1}^{p}=0.292 \mathrm{kPa}, C_{2}^{p}=$ $0.321, C_{3}^{p}=-0.260, C_{4}^{p}=0.201, C_{1}^{a}=-3.870 \mathrm{kPa}, C_{2}^{a}=4.830 \mathrm{kPa}, C_{3}^{a}=2.512 \mathrm{kPa}$ and $C_{4}^{a}=0.951 \mathrm{kPa}$. Our beating tension $T^{(0)}$ is adapted in order to satisfy the equibiaxial experimental results found by Lin and Yin [7]. The best agreement is obtained for $T^{(0)}=0.6 \mathrm{kPa}$ (see Fig. 4).

Since the exact displacements solutions are linear for such mechanical problems and the pressure is constant, they must be equal to their numerical FE approximations on each element with trilinear Lagrange interpolation. Therefore we chose to use only one element with 25 degree of freedom. All the stresses presented in the numerical 


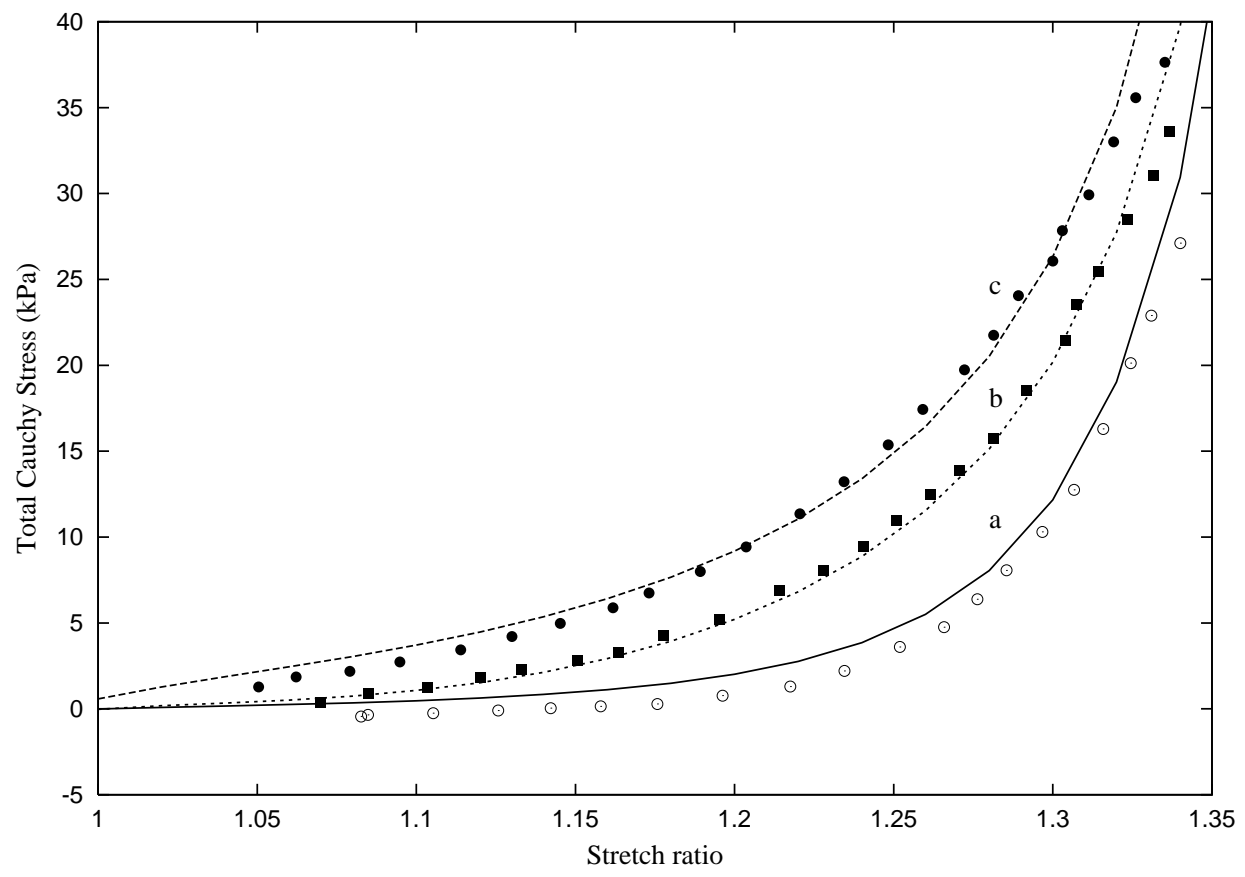

FIGURE 4. Equibiaxial tests: comparison with experimental data in passive case $(\beta=0$, curve a) and in active cases $(\beta=1)$ in the fiber direction (curve c) and the cross-fiber direction (curve b). Solid lines represent the computed FE solutions, symbols represent the experimental data $[7]$.

results are the total physical Cauchy stresses. In this case the $L^{2}$ norm of the error (between the exact and the FE numerical solution) is less than $10^{-12}$.

\subsection{Loading of an active thick-walled cylinder}

We simulate the mechanical behaviour of an active artery under physiological blood pressure $P_{\text {int }}$. This artery is modelised by a thick-walled cylinder with internal radius $R_{\mathrm{int}}=2 \mathrm{~mm}$, external radius $R_{\text {ext }}=3.5 \mathrm{~mm}$ and height $L=2 \mathrm{~cm}$. We assume that the medium is made of a hyperelastic anisotropic material with fibers oriented in the circumferential direction. In this study we used the strain-energy function suggested by Taber [16]:

$$
\begin{aligned}
W_{\mathrm{pas}}\left(\mathbf{E}_{P H}\right) & =\frac{a}{b}\left(e^{b\left(I_{1}-3\right)}-1\right), \\
W_{\mathrm{act}}^{f}\left(E_{P H}\right) & =\frac{a_{f}}{b_{f}}\left(e^{b_{f}\left(I_{4}-1\right)}-1-b_{f}\left(I_{4}-1\right)\right)+\frac{c_{f}}{d_{f}}\left(I_{4}+\frac{1}{I_{4}}-2\right)^{d_{f}} .
\end{aligned}
$$

Notice that he first derivative (with respect to $\left.I_{1}\right)$ of the passive energy $W_{\text {pas }}$ is not zero at rest $\left(I_{1}=3, I_{4}=1\right.$, $\beta=0$ ). The residual term, proportional to $\mathbf{I}$, is actually incorporated in the Lagrangian term $-p_{C} \mathbf{I}$ and this ensures the zero-stress conditions for the passive muscle free of loading, as in the previous case (sample of myocardium). The coefficients involved in this strain-energy function are $a=10 \mathrm{kPa}, b=0.1, a_{f}=10 \mathrm{kPa}$, $b_{f}=0.1, c_{f}=55 \mathrm{kPa}, d_{f}=1.8$ and $T^{(0)}=15 \mathrm{kPa}$. 


\subsubsection{Mechanical continuum approach}

The kinematics of the deformation for this loading case are $r=r(R), \theta=\Theta, z=\lambda Z$, where $\lambda$ is the stretch ratio in the $z$-direction. The non-zero components of the Cauchy stress tensor are

$$
\begin{aligned}
\tau^{r r} & =-p_{C}+2\left(\frac{R}{\lambda r}\right)^{2} \frac{\partial W}{\partial I_{1}} \\
\tau^{\theta \theta} & =-\frac{p_{C}}{r^{2}}+2 \frac{1}{R^{2}}\left(\frac{\partial W}{\partial I_{1}}+\frac{\partial W}{\partial I_{4}}\right)+\frac{\beta(t) T^{(0)}}{r^{2}} \\
\tau^{z z} & =-p_{C}+2 \lambda^{2} \frac{\partial W}{\partial I_{1}}
\end{aligned}
$$

These quantities verify:

$$
\begin{aligned}
\frac{\partial \tau^{r r}}{\partial r}+\frac{1}{r} \tau^{r r}-r \tau^{\theta \theta} & =0 \quad \text { (local equilibrium) } \\
\lambda \frac{r}{R} \frac{\partial r}{\partial R} & =1 \quad \text { (incompressibility) }
\end{aligned}
$$

with the boundary conditions $\tau^{r r}\left(r_{e}\right)=0, \tau^{r r}\left(r_{\text {int }}\right)=-P_{\text {int }}$ and $\tau^{z z}( \pm L / 2)=0$. The Cauchy stress components in the $\theta^{\alpha}$ direction are noted $\tau^{\alpha \alpha}$. The strain energy $W=W_{\text {pas }}+\beta(t) W_{\text {act }}^{f}$ is given by equations $((10)-(11))$. The two invariants $I_{1}$ and $I_{4}$ verify the relations

$$
I_{1}=\left(\frac{R}{\lambda r}\right)^{2}+\left(\frac{r}{R}\right)^{2}+\lambda^{2}, \quad I_{4}=\left(\frac{r}{R}\right)^{2} .
$$

The unknowns of the previous problem are $p_{C}(R), \lambda, r(R)$ and the solution of our nonlinear system of equations ((12)-(13)-(14)) are found numerically with a very high accuracy by using a Newton-Raphson method. We use this solution to validate the FE approximation.

\subsubsection{Finite element solution for particular loading}

\section{a. Artery with constant tonus}

In this simulation, the active fiber tension $\beta(t) T^{(0)}$ is kept constant with $\beta=0.5$. We use a pulsatile blood pressure given by $P_{\text {int }}=13+5 \sin (2 \pi t)$. The variations of the thick-walled cylinder radii are presented in Figure 5. Because the elastic properties of the arterial wall, as well as the active fiber tension, are not timedependent, the temporal evolution of the found internal and external radii (noted respectively $r_{\text {int }}$ and $r_{\text {ext }}$ ) are in phase with the pulsatile blood pressure.

Due to the symmetry of the problem, we used a quarter of an artery $(0 \leq \Theta \leq \pi / 2)$ with appropriate boundary conditions. A good agreement between the previous reference solution and the computed ones is obtained for six elements in the radial direction, and one element in the $\Theta$ and $Z$ directions.

\section{b. Artery with pressure-dependent tonus}

In 1902, Bayliss suggested that the distension of the vessel by blood pressure could act as a mechanical stimulus to the vascular smooth muscle cells, thereby contributing to their tone [1]. However, conclusive experimental support for this concept was available only recently. We now know that the degree of vascular distension appears to be a factor of importance in determining vascular tone. We used the suggested constitutive law to model a hypothetical autoregulation mechanism. 

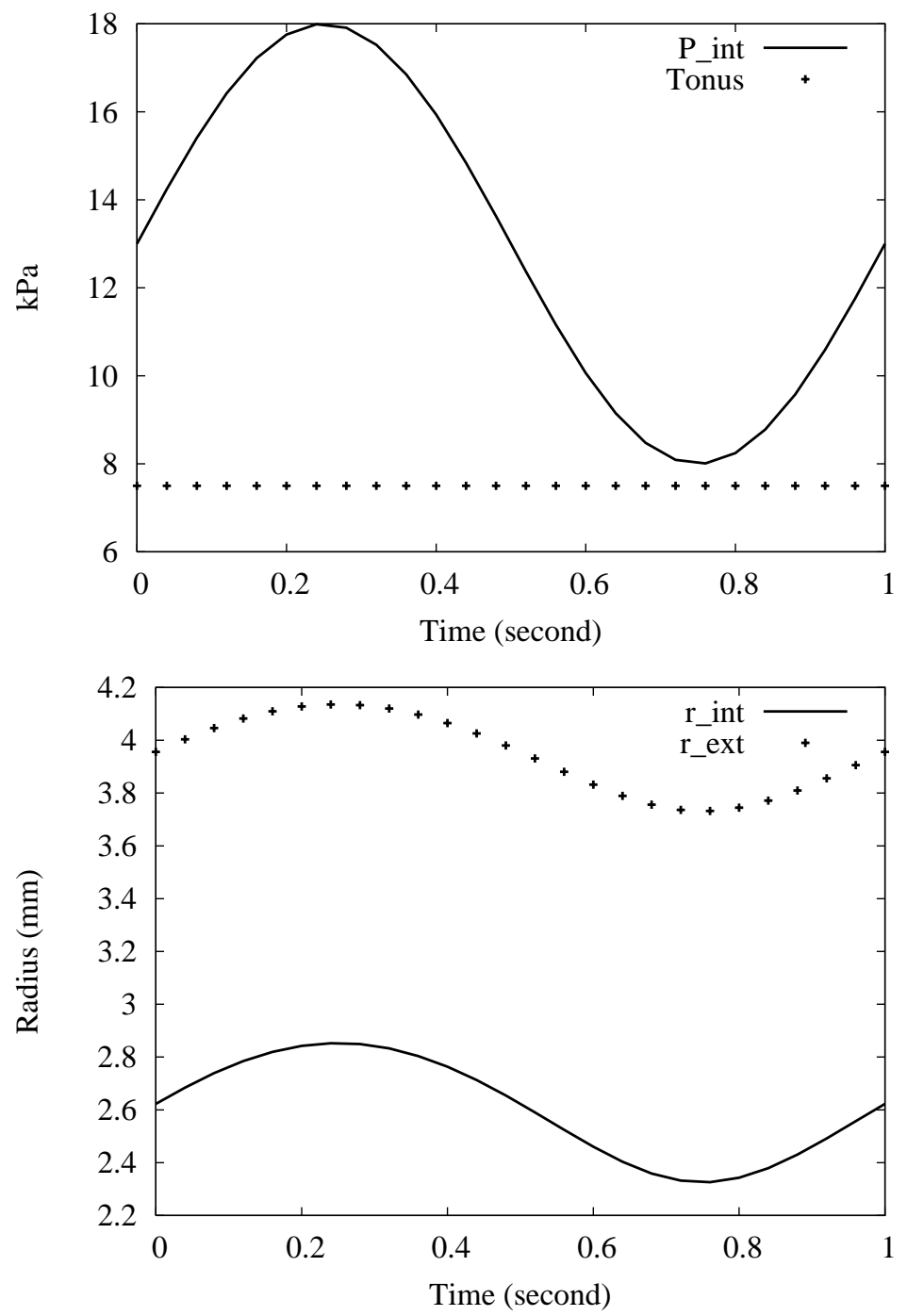

FiguRE 5. Temporal evolution of the internal $\left(r_{\text {int }}\right)$ and external $\left(r_{\text {ext }}\right)$ radii under a pulsatile blood pressure loading $P_{\text {int }}$ with a constant active fiber tension $\beta T^{(0)}$.

For this simulation, the active fiber tension as well as the rheological change are in phase with the pulsatile pressure, and we use as input data the following functions: $\beta(t)=0.5(1+\sin (2 \pi t))$ with $P_{\text {int }}=13+5 \sin (2 \pi t)$. The resulting variations of the thick-walled cylinder radii are presented in Figure 6 for this autoregulation law based on fluid pressure. The autoregulation is defined as the relationship between the activation function $\beta(t)$ and the pulsatile blood pressure $P_{\text {int }}$. Very interestingly, the results show that the kinematics of the arterial wall may be more sensitive to the change of mechanical properties than to the blood pressure. In other words, it appears that the internal and external radii increase when the blood pressure decreases. In fact, during this decrease of pressure, we assume that the material becomes more compliant. Thus, the wall kinematics is mainly driven by the change of rheology. Furthermore, although the pressure and activation are in phase, you can create with this autoregulation law some delay in the kinematic response. Therefore we believe that the pressure-activation interaction is a fundamental mechanism which must be well modeled to describe accurately the behaviour of the arterial wall under physiological or pathological conditions $[15,17]$. 

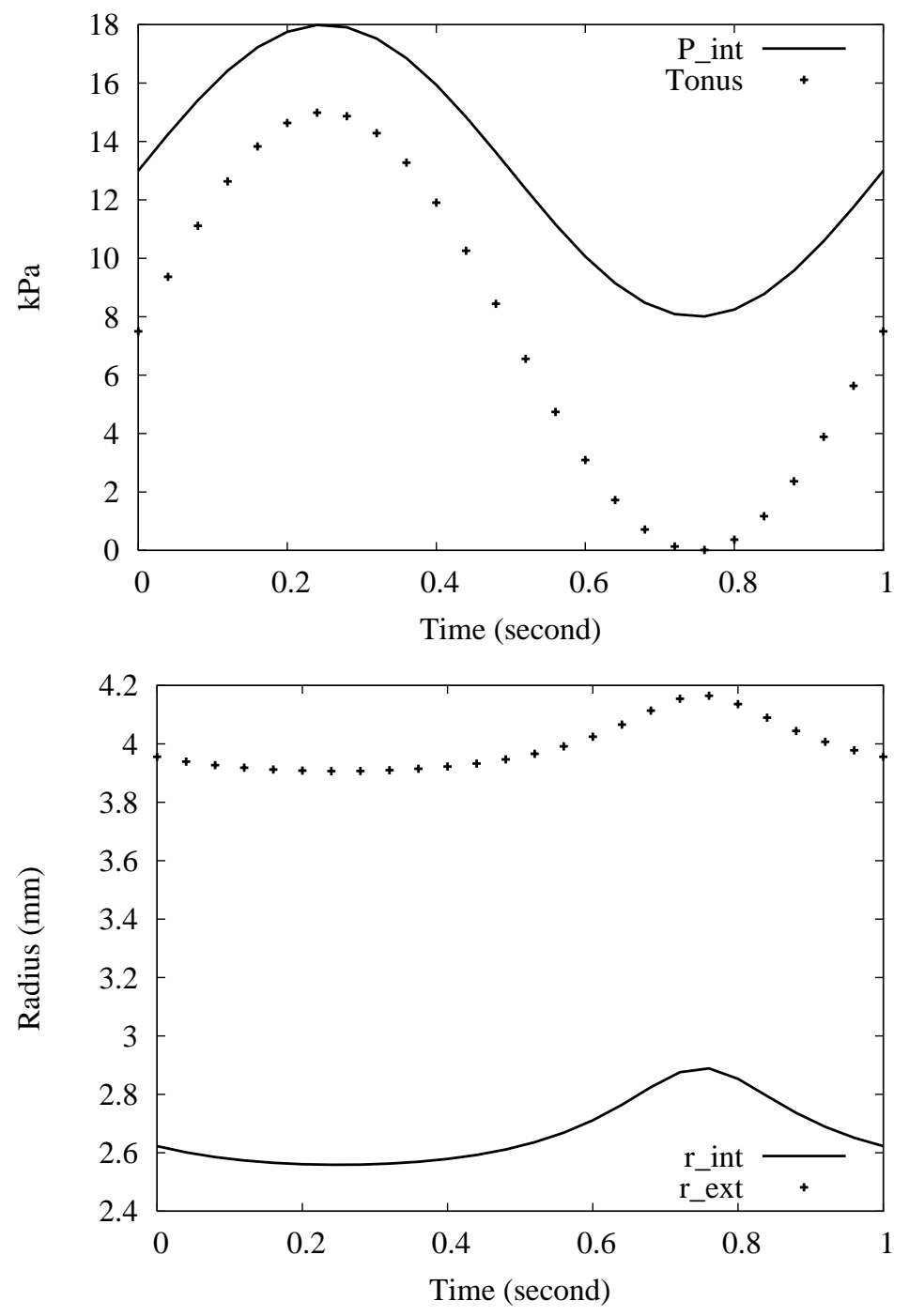

FiguRE 6 . Temporal evolution of the internal $\left(r_{\text {int }}\right)$ and external $\left(r_{\text {ext }}\right)$ radii under a pulsatile blood pressure loading $P_{\text {int }}$ with a time-dependant tonus $\beta T^{(0)}$.

\section{Conclusion}

A hyperelastic constitutive law, for use in anatomically accurate finite element models of living structures, is suggested for the passive and the active mechanical properties of incompressible biological tissues. This law considers the passive and active states as a same hyperelastic continuum medium, and uses an active tension in order to model the beating kinematic. The variational and the FE formulations are also presented, and the FE code has been successfully tested by comparing the numerical and the exact solutions for several quasi-static equilibrium problems. Such a code has been developed for finite elasticity and may be useful to a variety of applications in soft tissue biomechanics, such as pathological blood vessels with hypertension. This numerical tool may be adapted to three-dimensional large-scale problems such as modeling the heart or artery, by the use of an element by element method on a parallel computer. 


\section{Appendix A. Second Piola-Kirchoff stress tensor}

Using equations ((2)-(5)), we can write the components $P^{I J}$ of the second Piola-Kirchoff stress tensor $\mathbf{P}$ in the basis tensor $\mathbf{G}_{I}^{(x)} \otimes \mathbf{G}_{J}^{(x)}$ under the form:

$$
\begin{gathered}
P^{I J}=-p_{C} g^{(x) I J}+2 \delta^{I J} W_{1}+2 W_{4} f_{P}^{(x) I} f_{P}^{(x) J}+\beta(t) T^{(0)} f_{C}^{(x) I} f_{C}^{(x) J}, \\
\text { where } \quad W_{i}=\frac{\partial W}{\partial I_{i}}=\frac{\partial W_{\mathrm{pas}}}{\partial I_{i}}+\beta(t) \frac{\partial W_{\mathrm{act}}^{f}}{\partial I_{i}} \quad i=1,4
\end{gathered}
$$

$f_{P}^{(x) I}$ and $f_{C}^{(x) I}$ are the components of the unit vector $\mathbf{f}_{P}$ and $\mathbf{f}_{C}$ in the bases $\mathbf{G}_{I}^{(x)}$ and $\mathbf{g}_{I}^{(x)}$, respectively. The metric tensors $G^{(x) I J}, g^{(x) I J}$ are defined in Table 1.

Following the definition of the locally orthonormal body/fiber coordinate system we have $f_{P}^{(x) I}=\delta^{1 I}$. On the other hand the vector $\mathbf{f}_{C}$ is defined through:

$$
\mathbf{f}_{C}=\frac{\boldsymbol{\Phi}_{P C} \mathbf{f}_{P}}{\left\|\boldsymbol{\Phi}_{P C} \mathbf{f}_{P}\right\|}=\frac{\mathbf{f}_{P}^{(x) I} \mathbf{g}_{I}^{(x)}}{\left\|\mathbf{f}_{P}^{(x) I} \mathbf{g}_{I}^{(x)}\right\|}
$$

thus $f_{C}^{(x) I}=\frac{\delta^{1 I}}{\left\|\mathbf{g}_{1}^{(x)}\right\|}$ and we get finally:

$$
P^{I J}=-p_{C} g^{(x) I J}+2 \delta^{I J} W_{1}+2 W_{4} \delta^{1 I} \delta^{1 J}+\beta(t) T^{(0)} \frac{\delta^{1 I} \delta^{1 J}}{\left\|\mathbf{g}_{I=1}^{(x)}\right\|^{2}} .
$$

\section{Appendix B. Expression of THE SURFACE TRACTION S IN TERM OF THE PHYSICAL CAUCHY-STRESS TENSOR}

The surface traction per unit area of undeformable boundary $\mathcal{A}$ is given by $\mathbf{s}=\mathbf{J} \mathbf{N} \boldsymbol{\Phi}_{P C}^{-1} \boldsymbol{\tau}_{C}$ with $J=$ $\operatorname{det} \Phi_{P C}=1$ (incompressibility), $\mathbf{N}=N_{A}^{(\theta)} \mathbf{G}^{(\theta) A}$ (unit outward normal vector), $\boldsymbol{\tau}=\tau^{(\theta) p q} \mathbf{g}_{p}^{(\theta)} \mathbf{g}_{q}^{(\theta)}(\mathrm{Cauchy}$ stress tensor), and $\boldsymbol{\Phi}_{P C}=\mathbf{g}^{(\theta) \beta} \otimes \mathbf{G}_{\beta}^{(\theta)}$. Then

$$
\begin{aligned}
\mathbf{s} & =s^{\alpha} \mathbf{g}_{\alpha}^{(\theta)} \\
& =N_{A}^{(\theta)} \mathbf{G}^{(\theta) A} \mathbf{G}_{\beta}^{(\theta)} \otimes \mathbf{g}^{(\theta) \beta} \tau^{(\theta) \alpha^{\prime} \beta^{\prime}} \mathbf{g}_{\alpha^{\prime}}^{(\theta)} \otimes \mathbf{g}_{\beta^{\prime}}^{(\theta)} \\
& =N_{A}^{(\theta)} \mathbf{G}^{(\theta) A} \frac{\partial \Theta^{B}}{\partial \theta^{\beta}} \mathbf{G}_{B}^{(\theta)} \otimes \mathbf{g}^{(\theta) \beta} \tau^{(\theta) \alpha^{\prime} \beta^{\prime}} \mathbf{g}_{\alpha^{\prime}}^{(\theta)} \otimes \mathbf{g}_{\beta^{\prime}}^{(\theta)} \\
& =\left[N_{A}^{(\theta)} \frac{\partial \Theta^{A}}{\partial \theta^{\beta}} \tau^{(\theta) \alpha \beta}\right] \mathbf{g}_{\alpha}^{(\theta)},
\end{aligned}
$$

and so

$$
s^{\alpha}=N_{A}^{(\theta)} \frac{\partial \Theta^{A}}{\partial \theta^{\beta}} \tau^{(\theta) \alpha \beta}\left(=N_{I}^{(x)} \frac{\partial X^{I}}{\partial \theta^{\beta}} \tau^{(\theta) \alpha \beta}\right) .
$$

On the other hand, denoting by $\sigma^{(\theta) p q}$ the physical Cauchy stress components we have

$$
\sigma^{(\theta) p q}=\tau^{(\theta) p q}\left\|\mathbf{g}_{p}^{(\theta)}\right\|\left\|\mathbf{g}_{q}^{(\theta)}\right\|
$$

with no summation on $p, q$.

Equations ((18)-(19)) give together the expression of $s^{\alpha}$ in terms of the physical Cauchy-stress tensor components. 


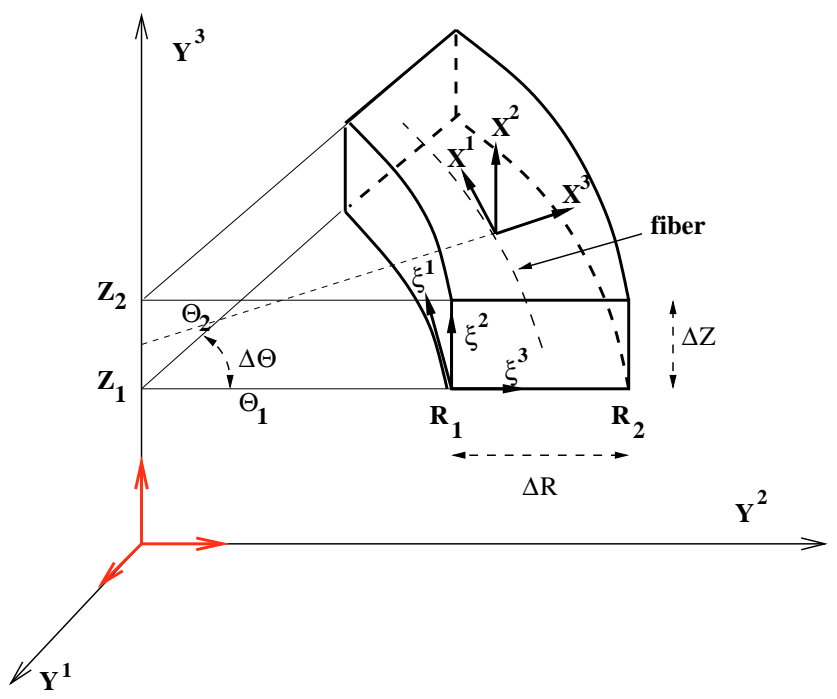

Figure 7. Cylindrical element.

\section{Appendix C. Finite Element formulation in the Cylindrical polar CASE}

The world coordinate system used here is $\left(\Theta^{A}\right)=(R, \Theta, Z)$, defined through $Y^{1}=R \cos \Theta, Y^{2}=R \sin \Theta$, $Y^{3}=Z$ (the same for $\left(\theta^{\alpha}\right)$ in the deformed configuration). The covariant metric tensor $\left(g^{(\theta) \alpha \beta}\right)$, for instance, is given by the diagonal matrix $\operatorname{diag}\left(1, r^{2}, 1\right)$. An element $e$ with size $\Delta R \times \Delta \Theta \times \Delta Z$ (see Fig. 7) is defined by

$$
R(\xi)=\Theta^{1}=R_{1}+\xi^{1} \Delta R, \quad \Theta(\xi)=\Theta^{2}=Y^{2}=\Theta_{1}+\xi^{2} \Delta \Theta, \quad Z(\xi)=\Theta^{3}=Z_{1}+\Delta Z \xi^{3} .
$$

In the following we use the relation $\frac{\partial}{\partial X^{I}}=(\cdot)_{, I}=\frac{\partial}{\partial \xi^{K}} \frac{\partial \xi^{K}}{\partial X^{I}}$, but $\frac{\partial \xi^{K}}{\partial X^{I}}=\frac{1}{a(I)} \delta_{I}^{K}$ with $a(1)=R(\xi) \Delta \Theta$, $a(2)=\Delta Z, a(3)=\Delta R$ and thus

$$
\frac{\partial}{\partial X^{I}}=(\cdot)_{, I}=\frac{1}{a(I)} \frac{\partial}{\partial \xi^{I}} \quad(\text { with no summation on } I)
$$

The covariant metric tensor $\left(G_{K L}^{(\xi)}\right)$ is given by the diagonal matrix $\operatorname{diag}\left(R^{2}(\Delta \Theta)^{2},(\Delta Z)^{2},(\Delta R)^{2}\right)$ and the volume element in equation (8) is $\mathrm{d} V=\sqrt{G_{K L}^{(\xi)}} \mathrm{d} \xi^{1} \mathrm{~d} \xi^{2} \mathrm{~d} \xi^{3}=a(1) a(2) a(3) \mathrm{d} \xi^{1} \mathrm{~d} \xi^{2} \mathrm{~d} \xi^{3}$.

\section{C.1. Expression of the left hand side (LHS) of equation (8)}

In the LHS of equation (8) we have, according to equation (17)

$$
\begin{aligned}
P^{I J} \Phi_{J}^{\cdot \alpha} & =P^{I J} \frac{\partial \theta^{\alpha}}{\partial X^{J}} \\
& =-p_{C} \frac{\partial X^{I}}{\partial \theta^{\beta}} g^{(\theta) \beta \alpha}+2 W_{1} \frac{\partial \theta^{\alpha}}{\partial X^{I}}+2 W_{4} \frac{\partial \theta^{\alpha}}{\partial X^{1}} \delta^{I 1}+\beta(t) T^{(0)} \frac{\frac{\partial \theta^{\alpha}}{\partial X^{1}}}{\frac{\partial \theta^{\beta}}{\partial X^{1}} \frac{\partial \theta^{\gamma}}{\partial X^{1}} g_{\beta \gamma}^{(\theta)}} \delta^{1 I} .
\end{aligned}
$$


- Terms $\frac{\partial \theta^{\alpha}}{\partial X^{I}}$ of equation (22) - Using equations ((6)-(7)) and (21) for the element $e$ we get

$$
\frac{\partial \theta^{\alpha}}{\partial X^{I}}=\frac{1}{a(I)} \sum_{p=1}^{8} \frac{\partial \psi_{p}}{\partial \xi^{I}} \theta_{\Delta(p, e)}^{\alpha} \quad \text { with no summation on } I
$$

- Terms $\frac{\partial X^{I}}{\partial \theta^{\alpha}}$ of equation (22) - We have $\frac{\partial X^{I}}{\partial \theta^{\alpha}}=\frac{1}{2 \operatorname{det} \Phi_{J}^{\cdot \alpha}} \varepsilon_{I J K} \varepsilon_{\alpha \beta \gamma} \frac{\partial \theta^{\beta}}{\partial X^{J}} \frac{\partial \theta^{\gamma}}{\partial X^{K}}$. The incompressibility equation writes $\operatorname{det}\left(g_{I J}^{(x)}\right)=\operatorname{det}\left(g_{\alpha \beta}^{(\theta)}\right)\left(\operatorname{det} \Phi_{J}^{\cdot \alpha}\right)^{2}=1$. Since $\operatorname{det} \Phi_{J}^{\cdot \alpha}>0$ and $g^{(\theta)}=r^{2}=\left(\theta^{1}\right)^{2}$, we have $\operatorname{det}\left(\Phi_{J}^{\cdot \alpha}\right)=\frac{1}{\theta^{1}}$ and

Using (23) we get

$$
\frac{\partial X^{I}}{\partial \theta^{\alpha}}=\frac{1}{2} \varepsilon_{I J K} \varepsilon_{\alpha \beta \gamma} \theta^{1} \frac{\partial \theta^{\beta}}{\partial X^{J}} \frac{\partial \theta^{\gamma}}{\partial X^{K}}
$$

$$
\frac{\partial X^{I}}{\partial \theta^{\alpha}}=\frac{1}{2} \sum_{J, K=1}^{3} \varepsilon_{I J K} \frac{1}{a(J) a(K)} \sum_{\beta, \gamma=1}^{3} \sum_{p, q, r=1}^{8} \varepsilon_{\alpha \beta \gamma} \theta_{\Delta(p, e)}^{1} \theta_{\Delta(q, e)}^{\beta} \theta_{\Delta(r, e)}^{\gamma} \psi_{p} \frac{\partial \psi_{q}}{\partial \xi^{J}} \frac{\partial \psi_{r}}{\partial \xi^{K}} .
$$

Finally we get for the first part of the LHS of equation (8) (involving $\left.\left(\psi_{n(e)}\right)_{, I}\right)$, for all $\Delta=1, \cdots, \Delta_{\max }$ and, for instance, $\alpha=1$ or 3 :

$$
\begin{aligned}
L H S_{1}(\Delta, \alpha)= & \Delta R \Delta \Theta \Delta Z \sum_{e} \sum_{n(e)=1}^{8} \Omega_{\Delta}^{n(e)}\left\{-\frac{p_{C}(e)}{2 \Delta R \Delta \Theta \Delta Z} \sum_{p, q, r=1}^{8} \sum_{\beta, \gamma=1}^{3} \varepsilon_{\alpha \beta \gamma} A^{n(e) p q r} \theta_{\Delta(p, e)}^{\beta} \theta_{\Delta(q, e)}^{\gamma} \theta_{\Delta(r, e)}^{1}\right. \\
& +\int_{V_{e}} 2 W_{1}\left(I_{1}, I_{4}, t\right) R(\xi) \sum_{I=1}^{3} \sum_{p=1}^{8} \frac{1}{a(I)^{2}} \frac{\partial \psi_{n(e)}}{\partial \xi^{I}} \frac{\partial \psi_{p}}{\partial \xi^{I}} \theta_{\Delta(p, e)}^{\alpha} \mathrm{d} \xi^{1} \mathrm{~d} \xi^{2} \mathrm{~d} \xi^{3} \\
& +\int_{V_{e}} \frac{2}{R(\xi)(\Delta \Theta)^{2}} W_{4}\left(I_{1}, I_{4}, t\right) \sum_{p=1}^{8} \frac{\partial \psi_{n(e)}}{\partial \xi^{1}} \frac{\partial \psi_{p}}{\partial \xi^{1}} \theta_{\Delta(p, e)}^{\alpha} \mathrm{d} \xi^{1} \mathrm{~d} \xi^{2} \mathrm{~d} \xi^{3} \\
& \left.+\beta(t) T^{(0)} \int_{V_{e}} \frac{\partial \psi_{n(e)}}{\partial \xi^{1}} \frac{\sum_{p=1}^{8} \frac{\partial \psi_{p}}{\partial \xi^{1}} \theta_{\Delta(p, e)}^{\alpha}}{(R(\xi) \Delta \Theta)^{2} I_{4}} \mathrm{~d} \xi^{1} \mathrm{~d} \xi^{2} \mathrm{~d} \xi^{3}\right\}
\end{aligned}
$$

where we denote, for all $i, j, k, l \in\{1, \cdots, 8\}$

$$
A^{i j k l}=\int_{V_{e}} \sum_{I, J, K=1}^{3} \varepsilon_{I J K} \frac{\partial \psi_{i}}{\partial \xi^{I}} \frac{\partial \psi_{j}}{\partial \xi^{J}} \frac{\partial \psi_{k}}{\partial \xi^{K}} \psi_{l} \mathrm{~d} \xi^{1} \mathrm{~d} \xi^{2} \mathrm{~d} \xi^{3} .
$$

The terms $W_{1}$ and $W_{4}$ are given in equation (15), where

$$
I_{1}=g_{I J}^{(x)} G^{(x) I J}=g_{I I}^{(x)}=\frac{\partial y^{r}}{\partial X^{I}} \frac{\partial y^{r}}{\partial X^{I}}=\sum_{\beta=1}^{3} \sum_{I=1}^{3} \frac{1}{a(I)^{2}}\left(\frac{\partial \theta^{\beta}}{\partial \xi^{I}}\right)^{2} g_{\beta \beta}^{(\theta)},
$$

and

$$
I_{4}=g_{I J}^{(x)} f_{P}^{I} f_{p}^{J}=g_{11}^{(x)}=\frac{1}{(R(\xi) \Delta \Theta)^{2}} \sum_{\beta=1}^{3}\left(\frac{\partial \theta^{\beta}}{\partial \xi^{1}}\right)^{2} g_{\beta \beta}^{(\theta)},
$$


are discretized as above (we skip the details for the sake of legibility).

When $\alpha=1$, the second part of the LHS of equation (8) reduces to $-P^{I J} \Phi_{J}^{\cdot 2} \Gamma_{2 I}^{1} \psi_{n(e)}$, and when $\alpha=2$ it reduces to $-P^{I J}\left(\Phi_{J}^{1} \Gamma_{1 I}^{2}+\Phi_{J}^{2} \Gamma_{2 I}^{2}\right) \psi_{n(e)}$. It is zero for $\alpha=3$. These terms are discretized as above, using:

$$
\Gamma_{2 I}^{1}=-r \theta_{, I}=\frac{1}{a(I)} \theta^{1} \frac{\partial \theta^{2}}{\partial \xi^{I}}, \quad \Gamma_{1 I}^{2}=\frac{1}{r} \theta_{, I}=\frac{1}{a(I)} \frac{1}{\theta^{1}} \frac{\partial \theta^{2}}{\partial \xi^{I}}, \quad \Gamma_{2 I}^{2}=\frac{1}{r} r_{, I}=\frac{1}{a(I)} \frac{1}{\theta^{1}} \frac{\partial \theta^{1}}{\partial \xi^{I}} .
$$

\section{C.2. Expression of the right hand side (RHS) of equation (8)}

- Term $s^{\alpha}$-We write $s^{\alpha}$ under the form (see Appendix B)

$$
s^{\alpha}=\frac{\partial X^{I}}{\partial \theta^{\beta}} \tau^{\alpha \beta} N_{I}^{(x)} .
$$

- Term $\mathrm{d} A$ of the boundary of element $e$ - On each face of the element $e$ the outward normal vector $N$ has only one non zero component $N_{I}^{(x)}$. We note $A(I, e)$ the part of $\partial e \cap \partial V$ with outward normal vector in the $G^{(x) I}$ direction (it may be empty). The surface measure is

$$
\mathrm{d} A=\mathrm{d} X^{K} \mathrm{~d} X^{L}=\sqrt{\operatorname{det} G_{K^{\prime} L^{\prime}}^{(\xi)}} \sqrt{G^{(\xi) I I}} \mathrm{~d} \xi^{K} \mathrm{~d} \xi^{L}=R(\xi) \Delta R \Delta \Theta \Delta Z \sqrt{G^{(\xi) I I}} \mathrm{~d} \xi^{K} \mathrm{~d} \xi^{L} \quad(K \neq L \neq I),
$$

with no summation on $I$. The metric tensors $G_{K L}^{(\xi)}$ and $G^{(\xi) K L}$ are defined in Table 1. In the case of fibers in the $\xi^{1}$-direction we have simply $G^{(\xi) I I}=\frac{1}{a(I)^{2}}$. Then, using equation (24) we get

$$
R H S=\frac{1}{2} \sum_{\{e, e \cap \partial V \neq \emptyset\}} \sum_{n(e)=1}^{8} \Omega_{\Delta}^{n(e)} \sum_{I, J, K=1}^{3} \sum_{\beta, \gamma, \delta=1}^{3} \varepsilon_{I J K} \varepsilon_{\beta \gamma \delta} \int_{A(I, e)} \frac{\sigma^{\alpha \beta}}{\left\|\mathbf{g}_{\alpha}^{(\theta)}\right\|\left\|\mathbf{g}_{\beta}^{(\theta)}\right\|} \theta^{1} \frac{\partial \theta^{\gamma}}{\partial \xi^{J}} \frac{\partial \theta^{\delta}}{\partial \xi^{K}} N_{I}^{(x)} \mathrm{d} \xi^{K} \mathrm{~d} \xi^{L} .
$$

In the case of an internal pressure loading $\sigma^{33}=-P_{\text {int }}$, the expression of $R H S$ reduces to

$$
\begin{aligned}
R H S= & -\frac{1}{2} P_{\text {int }} \delta^{\alpha 1} \sum_{\{e, e \cap \partial V \neq \emptyset\}} \sum_{n(e)=1}^{8} \Omega_{\Delta}^{n(e)} \sum_{J, K=1}^{2} \sum_{\gamma, \delta=2}^{3} \sum_{p, q, r=1}^{8} \varepsilon_{3 J K} \varepsilon_{1 \gamma \delta} \theta_{\Delta(p, e)}^{1} \theta_{\Delta(q, e)}^{\gamma} \theta_{\Delta(r, e)}^{\delta} \\
& \times \int_{A(3, e)} \psi_{p} \frac{\partial \psi_{q}}{\partial \xi^{J}} \frac{\partial \psi_{r}}{\partial \xi^{K}} \psi_{n(e)} \mathrm{d} \xi^{1} \mathrm{~d} \xi^{2} .
\end{aligned}
$$

\section{C.3. Discretization of the incompressibility equation}

We use a uniform approximation for the pressure on each mesh, so the incompressibility equation (9) is $\int_{V_{e}}\left(\operatorname{det} g_{I J}^{(x)}-1\right) \sqrt{\operatorname{det} G_{K L}^{(\xi)}} d \xi^{1} d \xi^{2} d \xi^{3}=0$, or equivalently

$$
\int_{V_{e}}\left(\theta^{1} \operatorname{det} \Phi_{J}^{\cdot \alpha}-1\right) \sqrt{\operatorname{det} G_{K L}^{(\xi)}} \mathrm{d} \xi^{1} \mathrm{~d} \xi^{2} \mathrm{~d} \xi^{3}=0
$$

Using the formula $\operatorname{det} \Phi_{J}^{\cdot \alpha}=\varepsilon_{I J K} \frac{\partial \theta^{1}}{\partial X^{I}} \frac{\partial \theta^{2}}{\partial X^{J}} \frac{\partial \theta^{3}}{\partial X^{K}}$ and equation (21), we write equation (27) under the form

$$
\sum_{p, q, r, s=1}^{8} A^{p q r s} \theta_{\Delta(p, e)}^{1} \theta_{\Delta(q, e)}^{2} \theta_{\Delta(r, e)}^{3} \theta_{\Delta(s, e)}^{1}=\left(R_{1}(e)+\frac{\Delta R}{2}\right) \Delta R \Delta \Theta \Delta Z=\operatorname{vol}\left(V_{e}\right)
$$

with $A^{\text {pqrs }}$ defined by (26). 


\section{REFERENCES}

[1] W.M. Bayliss, On the local reaction of the arterial wall to changes of internal pressure. J. Physiol. London 28 (1902) $220-231$.

[2] J. Berntsen, T.O. Espelid and A. Genz, Algorithm 698: DCUHRE: An adaptive multidimensional integration routine for a vector of integrals. ACM Trans. Math. Softw. 17 (1991) 452-456.

[3] P.H.M. Bovendeerd, T. Arts, J.M. Huyghe, D.H. van Campen and R.S. Reneman, Dependance of local left ventricular wall mechanics on myocardial fiber orientation: a model study. J. Biomech. 25 (1992) 1129-1140.

[4] P.G. Ciarlet, The finite element method for elliptic problems, Vol. 4 of Studies in Mathematics and its Applications. NorthHolland, Amsterdam-New York (1980).

[5] K.D. Costa, P.J. Hunter, J.S. Wayne, L.K. Waldman, J.M. Guccione and A.D. McCulloch, A three-dimensional finite element method for large elastic deformations of ventricular myocardium: Part I. Cylindrical and spherical polar coordinates. ASME J. Biomech. Eng. 118 (1996) 452-463.

[6] R. Glowinski and P. LeTallec, Augmented lagrangian and operator-splitting methods in nonlinear mechanics. SIAM, Philadelphia, PA (1989).

[7] D.H.S. Lin and F.C.P. Yin, A multiaxial constitutive law for mammalian left ventricular myocardium in steady-state barium contracture or tetanus. J. Biomech. Eng. 120 (1998) 504-517.

[8] L.E. Malvern, Introduction to the mechanics of a continuous medium. Prentice-Hall (1969).

[9] A.D. McCulloch, L.K. Waldman, J. Rogers and J. Guccione, Large scale finite element analysis of the beating heart. Crit. Rev. Biomed. Eng. 20 (1992) 427-449.

[10] J.J. Morge, B.S. Garbow and K.E. Hillstrom, User Guide for MINPACK-1. Technical Report ANL-80-74, Argonne National Laboratory (March 1980).

[11] J.T. Oden, Finite elements of nonlinear continua. McGraw-Hill, New York (1972).

[12] J. Ohayon and R.S. Chadwick, Effects of collagen microstructure on the mechanics of the left ventricle. Biophys. J. 54 (1988) 1077-1088.

[13] M.J.D. Powell, A hybrid method for nonlinear equations, in Numerical methods for nonlinear algebraic equations, P. Rabinowitz Ed. Gordon and Breach, New York (1970) 87-114.

[14] A. Quarteroni and A. Valli, Numerical approximation of partial differential equations, Vol. 23 of Springer Series in Computational Mathematics. Springer Verlag, Berlin (1994).

[15] G.M. Rubanyi, Mechanoreception by the vascular wall. Futura Publishing Company, Inc. (1993).

[16] L.A. Taber, On a nonlinear theory for muscle shells: Part II. Application to the beating left ventricle. J. Biomech. Eng. 113 (1991) 63-71.

[17] P. Teppaz, J. Ohayon and R. Herbin, Interaction fluide-structure active : écoulement artériel. C.R. Acad. Sci. Paris 324 (1997) 37-45.

[18] T.P. Usyk, Omens J.H. and A.D. McCulloch, Regional septal dysfunction in a three-dimensional computational model of focal myofiber dissaray. Am. J. Physiol. Heart Circ. Physiol. 281 (2001) 506-514.

[19] J. Zhang and C. Xu, A class of indefinite dogleg path mehods for unconstrained minimization. SIAM J. Optim. 9 (1999) 646-667.

To access this journal online:

www.edpsciences.org 